\title{
A NEW HILBERT-TYPE INTEGRAL INEQUALITY AND THE EQUIVALENT FORM
}

\author{
YONGJIN LI, JING WU, AND BING HE
}

Received 30 April 2006; Revised 10 August 2006; Accepted 21 August 2006

We give a new Hilbert-type integral inequality with the best constant factor by estimating the weight function. And the equivalent form is considered.

Copyright (c) 2006 Hindawi Publishing Corporation. All rights reserved.

\section{Introduction}

If $f, g$ are real functions such that $0<\int_{0}^{\infty} f^{2}(x) d x<\infty$ and $0<\int_{0}^{\infty} g^{2}(x) d x<\infty$, then we have (see [1])

$$
\int_{0}^{\infty} \int_{0}^{\infty} \frac{f(x) g(y)}{x+y} d x d y<\pi\left\{\int_{0}^{\infty} f^{2}(x) d x \int_{0}^{\infty} g^{2}(x) d x\right\}^{1 / 2}
$$

where the constant factor $\pi$ is the best possible. Inequality (1.1) is the well-known Hilbert's inequality. And inequality (1.1) had been generalized by Hardy in 1925 as follows.

$$
\begin{aligned}
& \text { If } f, g \geq 0, p>1,1 / p+1 / q=1,0<\int_{0}^{\infty} f^{p}(x) d x<\infty \text {, and } 0<\int_{0}^{\infty} g^{q}(x) d x<\infty \text {, then } \\
& \int_{0}^{\infty} \int_{0}^{\infty} \frac{f(x) g(y)}{x+y} d x d y<\frac{\pi}{\sin (\pi / p)}\left\{\int_{0}^{\infty} f^{p}(x) d x\right\}^{1 / p}\left\{\int_{0}^{\infty} g^{q}(x) d x\right\}^{1 / q}, \\
& \int_{0}^{\infty}\left(\int_{0}^{\infty} \frac{f(x)}{x+y} d x\right)^{p} d y<\left[\frac{\pi}{\sin (\pi / p)}\right]^{p} \int_{0}^{\infty} f^{p}(x) d x,
\end{aligned}
$$

where the constant factor $\pi / \sin (\pi / p)$ is the best possible. When $p=q=2$, (1.2) reduces to (1.1), inequality (1.2) is named of Hardy-Hilbert integral inequality, which is important in analysis and its applications. It has been studied and generalized in many directions by a number of mathematicians. 
In this paper, we give a new type of Hilbert's integral inequality as follows:

$$
\int_{0}^{\infty} \int_{0}^{\infty} \frac{f(x) g(y)}{x+y+\max \{x, y\}} d x d y<c\left\{\int_{0}^{\infty} f^{2}(x) d x \int_{0}^{\infty} g^{2}(x) d x\right\}^{1 / 2},
$$

where $c=\sqrt{2}(\pi-2 \arctan \sqrt{2})=1.7408 \ldots$

\section{Main results}

Lemma 2.1. Suppose $\varepsilon>0$, then

$$
\int_{1}^{\infty} x^{-\varepsilon-1} \int_{0}^{x^{-1}} \frac{1}{1+t+\max \{1, t\}} t^{(-1-\varepsilon) / 2} d t d x=O(1)\left(\varepsilon \rightarrow 0^{+}\right) .
$$

Proof. There exists $n \in \mathbb{N}$ which is large enough, such that $1+(-1-\varepsilon) / 2>0$ for $\varepsilon \in$ $(0,1 / n]$, we have

$$
\int_{0}^{x^{-1}} \frac{1}{1+t+\max \{1, t\}} t^{(-1-\varepsilon) / 2} d t<\int_{0}^{x^{-1}} t^{(-1-\varepsilon) / 2} d t=\frac{1}{1+(-1-\varepsilon) / 2}\left(\frac{1}{x}\right)^{1+(-1-\varepsilon) / 2} .
$$

Since for $a \geq 1$ the function $g(y)=\left(1 / y a^{y}\right)(y \in(0, \infty))$ is decreasing, we find

$$
\frac{1}{1+(-1-\varepsilon) / 2}\left(\frac{1}{x}\right)^{1+(-1-\varepsilon) / 2} \leq \frac{1}{1+((-1-1) / n) / 2}\left(\frac{1}{x}\right)^{1+((-1-1) / n) / 2},
$$

so

$$
\begin{aligned}
0 & <\int_{1}^{\infty} x^{-\varepsilon-1} \int_{0}^{x^{-1}} \frac{1}{1+t+\max \{1, t\}} t^{(-1-\epsilon) / 2} d t d x \\
& <\int_{1}^{\infty} x^{-1} \frac{1}{1+((-1-1) / n) / 2}\left(\frac{1}{x}\right)^{1+((-1-1) / n) / 2} d x \\
& =\left(\frac{1}{1+((-1-1) / n) / 2}\right)^{2} .
\end{aligned}
$$

Hence the relation (2.1) is valid. The lemma is proved.

Now we study the following inequality.

Theorem 2.2. Suppose $f(x), g(x) \geq 0,0<\int_{0}^{\infty} f^{2}(x) d x<\infty, 0<\int_{0}^{\infty} g^{2}(x) d x<\infty$. Then

$$
\int_{0}^{\infty} \int_{0}^{\infty} \frac{f(x) g(y)}{x+y+\max \{x, y\}} d x d y<c\left\{\int_{0}^{\infty} f^{2}(x) d x \int_{0}^{\infty} g^{2}(x) d x\right\}^{1 / 2}
$$

where the constant factor $c=\sqrt{2}(\pi-2 \arctan \sqrt{2})=1.7408 \ldots$ is the best possible. 
Proof. By Hölder's inequality, we have

$$
\begin{aligned}
& \int_{0}^{\infty} \int_{0}^{\infty} \frac{f(x) g(y)}{x+y+\max \{x, y\}} d x d y \\
&= \int_{0}^{\infty} \int_{0}^{\infty}\left[\frac{f(x)}{(x+y+\max \{x, y\})^{1 / 2}}\left(\frac{x}{y}\right)^{1 / 4}\right] \\
& \times\left[\frac{g(y)}{(x+y+\max \{x, y\})^{1 / 2}}\left(\frac{y}{x}\right)^{1 / 4}\right] d x d y \\
& \leq \int_{0}^{\infty} \int_{0}^{\infty} \frac{f^{2}(x)}{x+y+\max \{x, y\}}\left(\frac{x}{y}\right)^{1 / 2} d x d y \\
& \quad \times \int_{0}^{\infty} \int_{0}^{\infty} \frac{g^{2}(y)}{x+y+\max \{x, y\}}\left(\frac{y}{x}\right)^{1 / 2} d x d y .
\end{aligned}
$$

Define the weight function $\omega(u)$ as

$$
\omega(u):=\int_{0}^{\infty} \frac{1}{u+v+\max \{u, v\}}\left(\frac{u}{v}\right)^{1 / 2} d v
$$

then the above inequality yields

$$
\begin{aligned}
& \int_{0}^{\infty} \int_{0}^{\infty} \frac{f(x) g(y)}{x+y+\max \{x, y\}} d x d y \\
& \quad \leq\left[\int_{0}^{\infty} \omega(x) f^{2}(x) d x\right]^{1 / 2}\left[\int_{0}^{\infty} \omega(y) g^{2}(y) d y\right]^{1 / 2} .
\end{aligned}
$$

For fixed $u$, let $v=u t$, we have

$$
\begin{aligned}
\omega(u) & :=\int_{0}^{\infty} \frac{1}{1+t+\max \{1, t\}}\left(\frac{1}{t}\right)^{1 / 2} d t \\
& =\int_{0}^{1} \frac{1}{2+t}\left(\frac{1}{t}\right)^{1 / 2} d t+\int_{1}^{\infty} \frac{1}{1+2 t}\left(\frac{1}{t}\right)^{1 / 2} d t \\
& =\sqrt{2}(\pi-2 \arctan \sqrt{2}) .
\end{aligned}
$$

Thus

$$
\begin{aligned}
& \int_{0}^{\infty} \int_{0}^{\infty} \frac{f(x) g(y)}{x+y+\max \{x, y\}} d x d y \\
& \quad \leq \sqrt{2}(\pi-2 \arctan \sqrt{2})\left\{\int_{0}^{\infty} f^{2}(x) d x\right\}^{1 / 2}\left\{\int_{0}^{\infty} g^{2}(x) d x\right\}^{1 / 2} .
\end{aligned}
$$


If (2.10) takes the form of the equality, then there exist constants $a$ and $b$, such that they are not all zero and

$$
\begin{gathered}
a \frac{f^{2}(x)}{x+y+\max \{x, y\}}\left(\frac{x}{y}\right)^{1 / 2}=b \frac{g^{2}(y)}{x+y+\max \{x, y\}}\left(\frac{y}{x}\right)^{1 / 2} \\
\text { a.e. on }(0, \infty) \times(0, \infty) .
\end{gathered}
$$

Then we have

$$
a x f^{2}(x)=b y g^{2}(y) \quad \text { a.e. on }(0, \infty) \times(0, \infty) .
$$

Hence we have

$$
a x f^{2}(x)=b y g^{2}(y)=\text { constant }=d \quad \text { a.e. on }(0, \infty) \times(0, \infty) .
$$

Without losing the generality, suppose $a \neq 0$, then we obtain $f^{2}(x)=d / a x$, a.e. on $(0, \infty)$, which contradicts the fact that $0<\int_{0}^{\infty} f^{2}(x) d x<\infty$. Hence $(2.10)$ takes the form of strict inequality; we get (2.5).

For $0<\varepsilon<1$, set $f_{\varepsilon}(x)=x^{(-\varepsilon-1) / 2}$, for $x \in[1, \infty) ; f_{\varepsilon}(x)=0$, for $x \in(0,1) . g_{\varepsilon}(y)=$ $y^{(-\varepsilon-1) / 2}$, for $y \in[1, \infty) ; g_{\varepsilon}(y)=0$, for $y \in(0,1)$. Assume that the constant factor $c=$ $\sqrt{2}(\pi-2 \arctan \sqrt{2})$ in $(2.2)$ is not the best possible, then there exists a positive number $K$ with $K<c$, such that $(2.5)$ is valid by changing $c$ to $K$. We have

$$
\int_{0}^{\infty} \int_{0}^{\infty} \frac{f(x) g(y)}{x+y+\max \{x, y\}} d x d y<K\left\{\int_{0}^{\infty} f^{2}(x) d x\right\}^{1 / 2}\left\{\int_{0}^{\infty} g^{2}(x) d x\right\}^{1 / 2}=\frac{K}{\varepsilon},
$$

since

$$
\int_{0}^{\infty} \frac{1}{1+t+\max \{1, t\}} t^{(-1-\varepsilon) / 2} d t=\sqrt{2}(\pi-2 \arctan \sqrt{2})+o(1) \quad\left(\varepsilon \longrightarrow 0^{+}\right)
$$

Setting $y=t x$, by $(2.1)$, we find

$$
\begin{aligned}
\int_{0}^{\infty} & \int_{0}^{\infty} \frac{f(x) g(y)}{x+y+\max \{x, y\}} d x d y \\
& =\int_{1}^{\infty} \int_{1}^{\infty} \frac{x^{(-\varepsilon-1) / 2} y^{(-\varepsilon-1) / 2}}{x+y+\max \{x, y\}} d x d y \\
& =\int_{1}^{\infty} \int_{x^{-1}}^{\infty} \frac{x^{(-\varepsilon-1) / 2}(t x)^{(-\varepsilon-1) / 2}}{1+t+\max \{1, t\}} d x d t \\
& =\int_{1}^{\infty} x^{-\varepsilon-1}\left(\int_{0}^{\infty} \frac{1}{1+t+\max \{1, t\}} t^{(-1-\varepsilon) / 2} d t-\int_{0}^{x^{-1}} \frac{1}{1+t+\max \{1, t\}} t^{(-1-\varepsilon) / 2} d t\right) d x \\
& =\frac{1}{\varepsilon}[\sqrt{2}(\pi-2 \arctan \sqrt{2})+o(1)] .
\end{aligned}
$$


Since, for $\varepsilon>0$ small enough, we have

$$
\sqrt{2}(\pi-2 \arctan \sqrt{2})+o(1)<K,
$$

thus we get $\sqrt{2}(\pi-2 \arctan \sqrt{2}) \leq K$, then $c \leq K$, which contradicts the hypothesis. Hence the constant factor $c$ in (2.5) is the best possible.

Theorem 2.3. Suppose $f \geq 0$ and $0<\int_{0}^{\infty} f^{2}(x) d x<\infty$. Then

$$
\int_{0}^{\infty}\left[\int_{0}^{\infty} \frac{f(x)}{x+y+\max \{x, y\}} d x\right]^{2} d y<c^{2} \int_{0}^{\infty} f^{2}(x) d x
$$

where the constant factor $c^{2}=2(\pi-2 \arctan \sqrt{2})^{2}=3.0305 \ldots$ is the best possible. Inequality (2.18) is equivalent to (2.5).

Proof. Setting $g(y)$ as

$$
\int_{0}^{\infty} \frac{f(x)}{x+y+\max \{x, y\}} d x, \quad y \in(0, \infty)
$$

then by (2.5), we find

$$
\begin{aligned}
0 & <\int_{0}^{\infty} g^{2}(y) d y \\
& =\int_{0}^{\infty}\left[\int_{0}^{\infty} \frac{f(x)}{x+y+\max \{x, y\}} d x\right]^{2} d y \\
& =\int_{0}^{\infty} \int_{0}^{\infty} \frac{f(x) g(y)}{x+y+\max \{x, y\}} d x d y \\
& \leq \sqrt{2}(\pi-2 \arctan \sqrt{2})\left\{\int_{0}^{\infty} f^{2}(x) d x\right\}^{1 / 2}\left\{\int_{0}^{\infty} g^{2}(y) d y\right\}^{1 / 2} .
\end{aligned}
$$

Hence we obtain

$$
0<\int_{0}^{\infty} g^{2}(y) d y \leq 2(\pi-2 \arctan \sqrt{2})^{2} \int_{0}^{\infty} f^{2}(x) d x<\infty .
$$

By (2.5), both (2.20) and (2.21) take the form of strict inequality, so we have (2.18).

On the other hand, suppose that (2.18) is valid. By Hölder's inequality, we find

$$
\begin{aligned}
\int_{0}^{\infty} & \int_{0}^{\infty} \frac{f(x) g(y)}{x+y+\max \{x, y\}} d x d y \\
& =\int_{0}^{\infty}\left[\int_{0}^{\infty} \frac{f(x)}{x+y+\max \{x, y\}} d x\right] g(y) d y \\
& \leq\left\{\int_{0}^{\infty}\left[\int_{0}^{\infty} \frac{f(x)}{x+y+\max \{x, y\}} d x\right]^{2} d y\right\}^{1 / 2}\left\{\int_{0}^{\infty} g^{2}(y) d y\right\}^{1 / 2} .
\end{aligned}
$$

Then by (2.18), we have (2.5). Thus (2.5) and (2.18) are equivalent. 
6 A new Hilbert-type integral inequality

If the constant $c^{2}=2(\pi-2 \arctan \sqrt{2})^{2}$ in (2.18) is not the best possible, by (2.22), we may get a contradiction that the constant factor $c$ in (2.5) is not the best possible. Thus we complete the proof of the theorem.

\section{Acknowledgment}

The authors would like to thank the anonymous referees for their suggestions and corrections.

\section{References}

[1] G. H. Hardy, J. E. Littlewood, and G. Pólya, Inequalities, Cambridge University Press, Cambridge, 1952.

Yongjin Li: Department of Mathematics, Sun Yat-Sen University, Guangzhou 510275, China

E-mail address: stslyj@mail.sysu.edu.cn

Jing Wu: Department of Statistics, Sun Yat-Sen University, Guangzhou 510275, China

E-mail address: wjjosie@hotmail.com

Bing He: Department of Mathematics, Sun Yat-Sen University, Guangzhou 510275, China

E-mail address: hzs314@163.com 


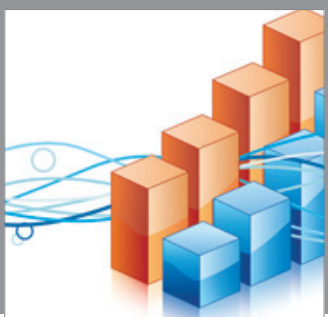

Advances in

Operations Research

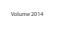

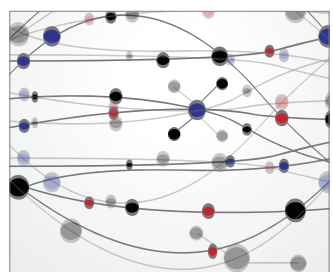

\section{The Scientific} World Journal
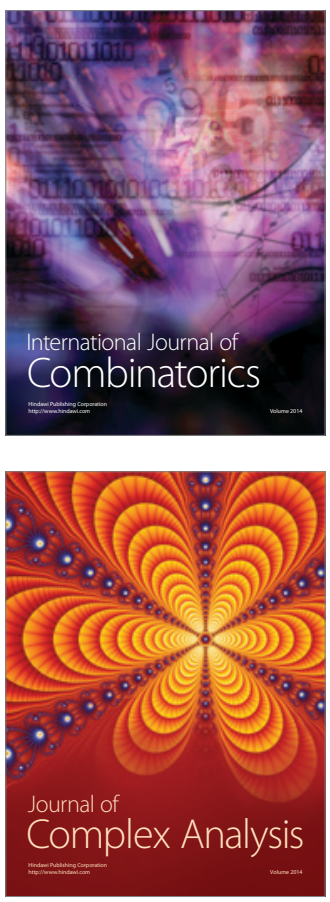

International Journal of

Mathematics and

Mathematical

Sciences
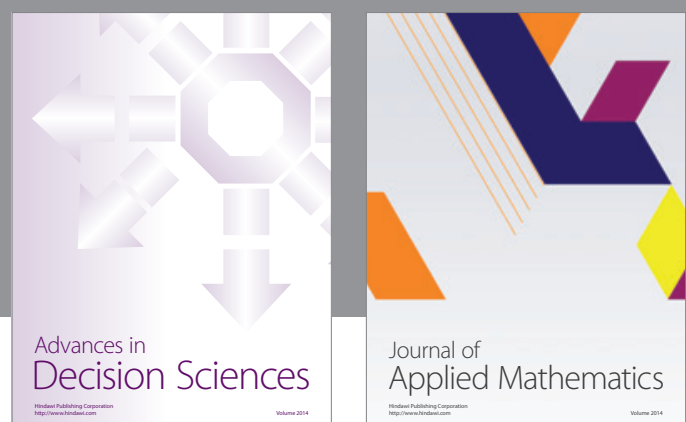

Journal of

Applied Mathematics
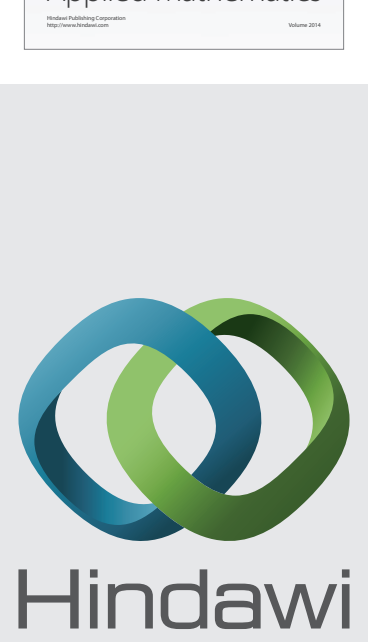

Submit your manuscripts at http://www.hindawi.com
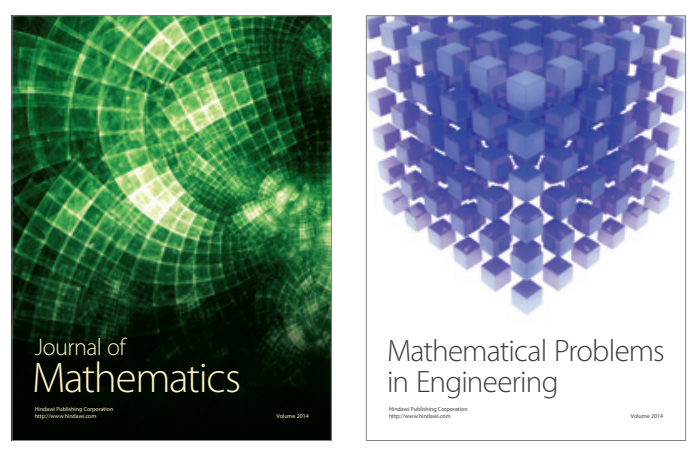

Mathematical Problems in Engineering
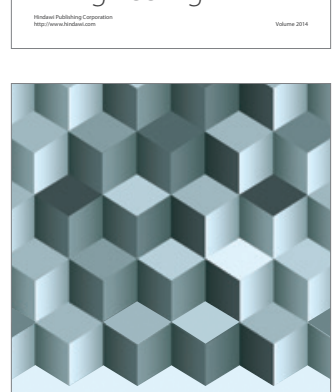

Journal of

Function Spaces
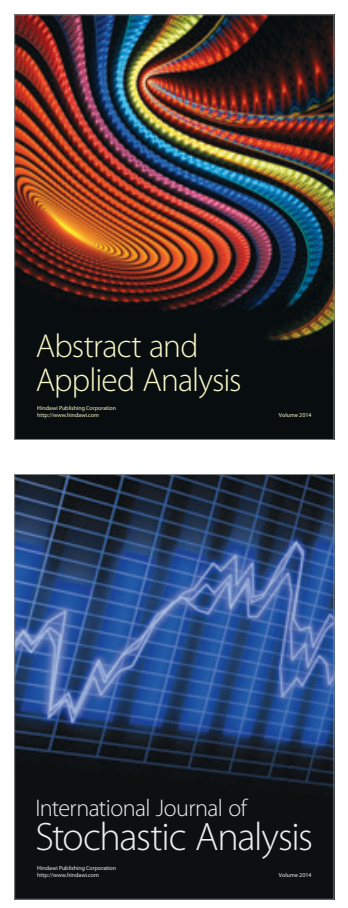

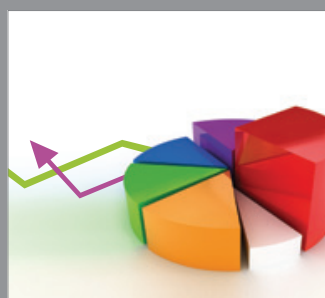

ournal of

Probability and Statistics

Promensencen
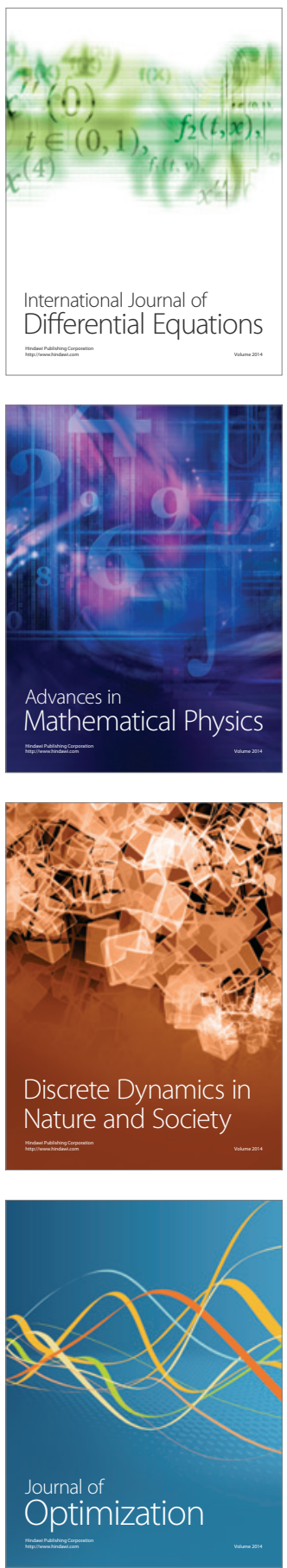